\title{
THREE PAIRS OF CONGRUENCES CONCERNING SUMS OF CENTRAL BINOMIAL COEFFICIENTS
}

\author{
GUO-SHUAI MAO AND ROBERTO TAURASO
}

\begin{abstract}
Recently the first author proved a congruence proposed in 2006 by Adamchuk: $\sum_{k=1}^{\left\lfloor\frac{2 p}{3}\right\rfloor}\left(\begin{array}{c}2 k \\ k\end{array}\right) \equiv 0\left(\bmod p^{2}\right)$ for any prime $p=1(\bmod 3)$. In this paper, we provide more examples (with proofs) of congruences of the same kind

$$
\sum_{k=1}^{\left\lfloor\frac{a p}{r}\right\rfloor}\left(\begin{array}{c}
2 k \\
k
\end{array}\right) x^{k} \quad\left(\bmod p^{2}\right)
$$

where $p$ is a prime such that $p \equiv 1(\bmod r), a / r$ is a fraction in $(1 / 2,1)$ and $x$ is a $p$-adic integer. The key ingredients are the $p$-adic Gamma functions $\Gamma_{p}$ and a special class of computer-discovered hypergeometric identities.
\end{abstract}

\section{INTRODUCTION}

In the past decades, many people studied congruences modulo a power of a prime $p$ for sums of binomial coefficients. A certain number concern the central binomial coefficients and have the form

$$
\sum_{k=0}^{n}\left(\begin{array}{c}
2 k \\
k
\end{array}\right) x^{k}
$$

where the upper limit $n$ is usually $p-1$ or $\frac{p-1}{2}$. We would like to mention a few of them that we will need later. By [9, p. 156] and [12, Theorem 1.1], for any prime $p>3$,

$$
\begin{aligned}
& \sum_{k=0}^{p-1}\left(\begin{array}{c}
2 k \\
k
\end{array}\right)(-2)^{k} \equiv 1-\frac{4 p q_{p}(2)}{3} \quad\left(\bmod p^{3}\right) \\
& \sum_{k=0}^{\frac{p-1}{2}} \frac{\left(\begin{array}{c}
2 k \\
k
\end{array}\right)}{(-32)^{k}} \equiv\left(\frac{2}{p}\right)\left(1+\frac{p q_{p}(2)}{6}-\frac{p^{2} q_{p}^{2}(2)}{8}\right) \quad\left(\bmod p^{3}\right)
\end{aligned}
$$

2010 Mathematics Subject Classification. 11A07, 05A10, 11B65, 33C20, 33B15.

Key words and phrases. Congruence; central binomial coefficient; $p$-adic gamma function; hypergeometric functions; harmonic numbers. 
where $q_{p}(a)=\frac{a^{p-1}-1}{p}$ is the so-called Fermat quotient. Moreover, by [10, Theorem 1.3], [12, Theorem 1.1], for any prime $p>5$,

$$
\begin{aligned}
& \sum_{k=0}^{p-1}\left(\begin{array}{c}
2 k \\
k
\end{array}\right)(-1)^{k} \equiv\left(\frac{p}{5}\right)\left(1-2 p f_{p}\right) \quad\left(\bmod p^{3}\right) \\
& \sum_{k=0}^{\frac{p-1}{2}} \frac{\left(\begin{array}{c}
2 k \\
k
\end{array}\right)}{(-16)^{k}} \equiv\left(\frac{p}{5}\right)\left(1+\frac{p f_{p}}{2}\right) \quad\left(\bmod p^{3}\right)
\end{aligned}
$$

where $f_{p}=\frac{F_{p-\left(\frac{p}{5}\right)}}{p}$ is the Fibonacci quotient and $F_{n}$ denotes the $n$th Fibonacci number.

Less common are the examples where the upper limit of the sum is strictly between $\frac{p-1}{2}$ and $p-1$. In 2014, Pan and Sun [10] proved that for any prime $p \equiv 1(\bmod 4)$,

$$
\sum_{k=0}^{\left\lfloor\frac{3 p}{4}\right\rfloor} \frac{\left(\begin{array}{c}
2 k \\
k
\end{array}\right)}{(-4)^{k}} \equiv\left(\frac{2}{p}\right) \quad\left(\bmod p^{2}\right)
$$

Recently Mao [6, 7] proved that for any prime $p \equiv 1(\bmod 3)$, we have

$$
\sum_{k=1}^{\left\lfloor\frac{2 p}{3}\right\rfloor}\left(\begin{array}{c}
2 k \\
k
\end{array}\right) \equiv 0 \quad \text { and } \quad \sum_{k=0}^{\left\lfloor\frac{5 p}{6}\right\rfloor} \frac{\left(\begin{array}{c}
2 k \\
k
\end{array}\right)}{16^{k}} \equiv\left(\frac{3}{p}\right) \quad\left(\bmod p^{2}\right) .
$$

The first congruence has been conjectured in 2006 by Adamchuk [1.

The main purpose of this paper is to show more congruences of the same kind.

Theorem 1. Let $p$ be a prime. If $p \equiv 1(\bmod 3)$ then

$$
\sum_{k=1}^{\left\lfloor\frac{2 p}{3}\right\rfloor}\left(\begin{array}{c}
2 k \\
k
\end{array}\right)(-2)^{k} \equiv 0, \quad \sum_{k=0}^{\left\lfloor\frac{5 p}{6}\right\rfloor} \frac{\left(\begin{array}{c}
2 k \\
k
\end{array}\right)}{(-32)^{k}} \equiv\left(\frac{2}{p}\right) \quad\left(\bmod p^{2}\right) .
$$

If $p \equiv 1(\bmod 5)$ then

$$
\sum_{k=1}^{\left\lfloor\frac{4 p}{5}\right\rfloor}\left(\begin{array}{c}
2 k \\
k
\end{array}\right)(-1)^{k} \equiv 0, \quad \sum_{k=1}^{\left\lfloor\frac{7 p}{10}\right\rfloor} \frac{\left(\begin{array}{c}
2 k \\
k
\end{array}\right)}{(-16)^{k}} \equiv 0 \quad\left(\bmod p^{2}\right),
$$

and

$$
\sum_{k=1}^{\left\lfloor\frac{3 p}{5}\right\rfloor}\left(\begin{array}{c}
2 k \\
k
\end{array}\right)(-1)^{k} \equiv 0, \quad \sum_{k=1}^{\left\lfloor\frac{9 p}{10}\right\rfloor} \frac{\left(\begin{array}{c}
2 k \\
k
\end{array}\right)}{(-16)^{k}} \equiv 0 \quad\left(\bmod p^{2}\right) .
$$

The first pair of congruences seems to have (so far) slipped the attention of the mathematical community whereas the other pairs appeared as conjectures in [12].

The paper is organized as follows. In Section 2 we recall some properties of the $p$-adic Gamma function and some known congruences involving harmonic sums. Section 3 is devoted to a auxiliary binomial sum defined as

$$
S_{m}(x)=\frac{1}{\left(\begin{array}{c}
2 m \\
m
\end{array}\right)} \sum_{k=0}^{m-1} \frac{\left(\begin{array}{c}
2 k \\
k
\end{array}\right) x^{k-m}}{k-m}
$$


It turns out that $S_{m}(x)$ can be evaluated modulo a prime for some special values of $m$ and $x$ by using a class of finite hypergeometric identites computer-discovered by Shalosh B. Ekhad (well-known co-author of Doron Zeilberger). In Section 4, we explain how $S_{m}(x)$ is related to our main results. Finally, we give a proof of Theorem 1 which reveals why the congruences (5), (6), and (7) come in pairs.

\section{Notations AND PRELIMINARIES}

The generalized hypergeometric function is defined as

$$
{ }_{m+1} F_{m}\left[\begin{array}{cccc}
\alpha_{0} & \alpha_{1} & \ldots & \alpha_{m} \\
& \beta_{1} & \ldots & \beta_{m}
\end{array} \mid z\right]=\sum_{k=0}^{\infty} \frac{\left(\alpha_{0}\right)_{k}\left(\alpha_{1}\right)_{k} \cdots\left(\alpha_{m}\right)_{k}}{\left(\beta_{1}\right)_{k} \cdots\left(\beta_{m}\right)_{k}} \cdot \frac{z^{k}}{k !},
$$

where $\alpha_{0}, \ldots, \alpha_{m}, \beta_{1}, \ldots, \beta_{m}, z \in \mathbb{C}$ and

$$
(\alpha)_{k}= \begin{cases}\alpha(\alpha+1) \cdots(\alpha+k-1) & \text { if } k \geq 1, \\ 1 & \text { if } k=0 .\end{cases}
$$

is the rising factorial or the Pochhammer symbol.

The $p$-adic gamma function $\Gamma_{p}$ is defined as the continuous extension to the ring of all $p$-adic integers $\mathbb{Z}_{p}$ of the sequence

$$
n \rightarrow(-1)^{n} \prod_{\substack{1 \leq j<n \\(k, p)=1}} k
$$

where $p$ is a prime and $n$ is a positive integer.

It follows that $\Gamma_{p}(0)=1$ and, for $x \in \mathbb{Z}_{p}$,

$$
\frac{\Gamma_{p}(x+1)}{\Gamma_{p}(x)}= \begin{cases}-x & \text { if }|x|_{p}=1 \\ -1 & \text { if }|x|_{p}>1\end{cases}
$$

where $|\cdot|_{p}$ is the $p$-adic norm. Throughout the whole paper, we will only use the most basic properties of $\Gamma_{p}$ (see [11, Chap. 7] as a general reference). In particular, we recall that (see for example [8, (3.2)]) for any $\alpha \in \mathbb{Z}_{p}$,

$$
\frac{\Gamma_{p}^{\prime}(x)}{\Gamma_{p}(x)} \equiv \Gamma_{p}^{\prime}(0)+H_{p-\langle-x\rangle_{p}-1} \quad(\bmod p)
$$

where $H_{n}=\sum_{k=1}^{n} \frac{1}{k}$ is the $n$th harmonic number and $\langle x\rangle_{p}$ is the least nonnegative residue of $x$ modulo $p$.

Lemma 1. For any prime $p>3$,

$$
\begin{aligned}
& H_{\left\lfloor\frac{p}{2}\right\rfloor} \equiv-2 q_{p}(2), \quad H_{\left\lfloor\frac{p}{4}\right\rfloor} \equiv-3 q_{p}(2) \quad(\bmod p), \\
& H_{\left\lfloor\frac{p}{3}\right\rfloor} \equiv-\frac{3 q_{p}(3)}{2}, \quad H_{\left\lfloor\frac{p}{6}\right\rfloor} \equiv-2 q_{p}(2)-\frac{3 q_{p}(3)}{2} \quad(\bmod p) .
\end{aligned}
$$


For any prime $p>5$,

$$
\begin{aligned}
& H_{\left\lfloor\frac{p}{5}\right\rfloor} \equiv-\frac{5 q_{p}(5)}{4}-\frac{5 f_{p}}{4}, \quad H_{\left\lfloor\frac{2 p}{5}\right\rfloor} \equiv-\frac{5 q_{p}(5)}{4}+\frac{5 f_{p}}{4}(\bmod p), \\
& H_{\left\lfloor\frac{p}{10}\right\rfloor} \equiv-2 q_{p}(2)-\frac{5 q_{p}(5)}{4}-\frac{15 f_{p}}{4}, \quad H_{\left\lfloor\frac{3 p}{10}\right\rfloor} \equiv-2 q_{p}(2)-\frac{5 q_{p}(5)}{4}+\frac{15 f_{p}}{4} \quad(\bmod p) .
\end{aligned}
$$

Proof. For a proof of the first group of congruences see [4, (41)-(44)]. As regards the second group, the first two congruences follow from

$$
H_{\left\lfloor\frac{2 p}{5}\right\rfloor}+H_{\left\lfloor\frac{p}{5}\right\rfloor} \equiv-\frac{5 q_{p}(5)}{2}, \quad H_{\left\lfloor\frac{2 p}{5}\right\rfloor}-H_{\left\lfloor\frac{p}{5}\right\rfloor} \equiv \frac{5 f_{p}}{2} \quad(\bmod p)
$$

see [5, (15)] and [16, (4.7)] respectively. Finally, the last two congruences are obtained by considering two more linear relations [14, (12) with $n=10]$,

$$
10\left(q_{p}(2)+q_{p}(5)\right) \equiv 10 q_{p}(10) \equiv-\sum_{k=1}^{9} H_{\left\lfloor\frac{k p}{10}\right\rfloor} \equiv-2 \sum_{k=1}^{4} H_{\left\lfloor\frac{k p}{10}\right\rfloor}-H_{\left\lfloor\frac{p}{2}\right\rfloor} \quad(\bmod p)
$$

and

$$
5 f_{p} \equiv 2 \sum_{k=1}^{\left\lfloor\frac{4 p}{5}\right\rfloor} \frac{(-1)^{k}}{k}=2 \sum_{k=1}^{\left\lfloor\frac{2 p}{5}\right\rfloor} \frac{1}{2 k}+2 \sum_{k=\left\lfloor\frac{p}{10}\right\rfloor+1}^{\frac{p-1}{2}} \frac{1}{2 k-p} \equiv H_{\left\lfloor\frac{2 p}{5}\right\rfloor}+H_{\left\lfloor\frac{p}{2}\right\rfloor}-H_{\left\lfloor\frac{p}{10}\right\rfloor} \quad(\bmod p)
$$

where the congruence on the left is given by [15, Theorem 2].

\section{An AUXILIARY SUM AND SOME SPECIAL EVALUATIONS}

The sum $S_{m}(x)$ introduced in the first section can be evaluated modulo a prime through a finite hypergeometric series ${ }_{2} F_{1}$ which depend on two parameters $\alpha$ and $\beta$ to be chosen suitably.

Theorem 2. Let $p$ be a prime, and let $\alpha, \beta \in \mathbb{Z}_{p}$ with $\beta$ prime to $p$. Then for any positive integer $m \leq \frac{p-1}{2}$,

$$
S_{m}(x) \equiv \frac{1}{\beta p}\left({ }_{2} F_{1}\left[\begin{array}{cc}
-m, & -\beta p \\
\frac{1}{2}-m-\alpha p
\end{array} ; \frac{1}{4 x}\right]-1\right) \quad(\bmod p) .
$$


Proof.

$$
\begin{aligned}
& \sum_{k=0}^{m-1} \frac{\left(\begin{array}{c}
2 k \\
k
\end{array}\right) x^{k}}{k-m}=-\frac{1}{m} \sum_{k=0}^{m-1} \frac{(-m)_{k}\left(\frac{1}{2}\right)_{k}(4 x)^{k}}{(1)_{k}(-m+1)_{k}} \\
& \equiv-\frac{1}{m} \sum_{k=0}^{m-1} \frac{(-m)_{k}\left(\frac{1}{2}+\alpha p\right)_{k}(4 x)^{k}}{(1)_{k}(-m+1+\beta p)_{k}} \quad(\bmod p) \\
& =-\frac{1}{m}\left({ }_{2} F_{1}\left[\begin{array}{cc}
-m, & \frac{1}{2}+\alpha p \\
-m+1+\beta p
\end{array} ; 4 x\right]-\frac{\left(\frac{1}{2}+\alpha p\right)_{m}(-4 x)^{m}}{(-m+1+\beta p)_{m}}\right) \\
& =-\frac{\left(\frac{1}{2}+\alpha p\right)_{m}(-4 x)^{m}}{m(-m+1+\beta p)_{m}}\left({ }_{2} F_{1}\left[\begin{array}{cc}
-m, & -\beta p \\
\frac{1}{2}-m-\alpha p
\end{array} ; \frac{1}{4 x}\right]-1\right) \\
& \equiv\left(\begin{array}{c}
2 m \\
m
\end{array}\right) \frac{x^{m}}{\beta p}\left({ }_{2} F_{1}\left[\begin{array}{cc}
-m, & -\beta p \\
\frac{1}{2}-m-\alpha p
\end{array} ; \frac{1}{4 x}\right]-1\right) \quad(\bmod p) \text {. }
\end{aligned}
$$

where we used the linear transformation [3, 15.8.6]

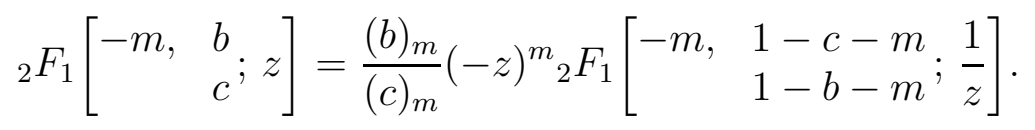

Some evaluations of $S_{m}(x)$ are straighforward. For $x=\frac{1}{4}$, we let $\alpha=0$ and $\beta=1$ and we find

$$
\begin{aligned}
S_{m}\left(\frac{1}{4}\right) & \equiv \frac{1}{p}\left({ }_{2} F_{1}\left[\begin{array}{cc}
-m, & -p \\
\frac{1}{2}-m & ; 1
\end{array}\right]-1\right)=\frac{1}{p}\left(\frac{\left(\frac{1}{2}-m+p\right)_{m}}{\left(\frac{1}{2}-m\right)_{m}}-1\right) \\
& =\frac{1}{p}\left(\prod_{j=1}^{m}\left(1-\frac{2 p}{2 j-1}\right)-1\right) \equiv-2 \sum_{j=1}^{m} \frac{1}{2 j-1}=H_{m}-2 H_{2 m} \quad(\bmod p),
\end{aligned}
$$

where we applied the Chu-Vandermonde identity [3, 15.4.24]

$$
{ }_{2} F_{1}\left[\begin{array}{cc}
-m, & b \\
c & ; 1
\end{array}\right]=\frac{(c-b)_{m}}{(c)_{m}} .
$$

Another easy case is for $m=\frac{p-1}{2}$,

$$
\begin{aligned}
S_{\frac{p-1}{2}}(x) & \equiv(-1)^{n} \sum_{k=0}^{n-1} \frac{\left(\begin{array}{l}
n \\
k
\end{array}\right)(-4)^{k} x^{k-n}}{k-n} \equiv-\sum_{k=0}^{n-1} \frac{\left(\begin{array}{l}
n \\
k
\end{array}\right)(-4 x)^{k-n}}{n-k} \\
& =-\sum_{k=1}^{n} \frac{\left(\begin{array}{l}
n \\
k
\end{array}\right)}{k(-4 x)^{k}} \equiv-\sum_{k=1}^{p-1} \frac{\left(\begin{array}{c}
2 k \\
k
\end{array}\right)}{k(16 x)^{k}} \quad(\bmod p) .
\end{aligned}
$$

Therefore, in view of [13], the following congruences hold modulo a prime $p$,

$$
S_{\frac{p-1}{2}}\left(\frac{1}{16}\right) \equiv 0, \quad S_{\frac{p-1}{2}}\left(-\frac{1}{32}\right) \equiv 4 q_{p}(2), \quad S_{\frac{p-1}{2}}\left(-\frac{1}{16}\right) \equiv 5 f_{p} .
$$


More evaluations can be made. In view of (9) and by using the Maple package twoFone written by Zeilberger [2], after a systematic search, we were able to obtain various evaluations of $S_{m}(x)$ collected in Table 1. In order to explain how the table works we present the full proof of two rows. The other rows can be verified along the same scheme.

TABLE 1 . Values of $S_{m}(x)$ modulo $p$ for $p \equiv 1(\bmod r)$

\begin{tabular}{|c|c|c|c|c|c|}
\hline$r$ & $m$ & $x$ & $\alpha, \beta$ & ${ }_{2} F_{1}$ & $S_{m}(x)$ \\
\hline 3 & $\frac{p-1}{6}$ & $\frac{1}{16}$ & $0, \frac{1}{2}$ & $(-3)^{3 m}$ & $q_{p}(3)$ \\
3 & $\frac{p-1}{6}$ & $-\frac{1}{32}$ & $-\frac{1}{6}, \frac{1}{3}$ & $(-3)^{3 m}$ & $\frac{3}{2} q_{p}(3)$ \\
3 & $\frac{p-1}{3}$ & 1 & $-1,-1$ & $\frac{2^{4 m}\left(\frac{3}{2}\right)_{2 m}}{3^{3 m}\left(\frac{4}{3}\right)_{2 m}}$ & $q_{p}(3)$ \\
3 & $\frac{p-1}{3}$ & -2 & $-1,-1$ & $\frac{2^{3 m}\left(\frac{3}{2}\right)_{2 m}}{3^{3 m}\left(\frac{5}{6}\right)_{m}\left(\frac{7}{6}\right)_{m}}$ & $-q_{p}(2)+\frac{3}{2} q_{p}(3)$ \\
\hline 4 & $\frac{p-1}{4}$ & $-\frac{1}{4}$ & $0, \frac{1}{2}$ & $\frac{2^{2 m}\left(\frac{3}{4}\right)_{m}}{\left(\frac{1}{2}\right)_{m}}$ & $\frac{3}{2} q_{p}(2)$ \\
\hline 5 & $\frac{p-1}{10}$ & $-\frac{1}{16}$ & $\frac{1}{2}, \frac{1}{2}$ & $\frac{5^{5 m}\left(\frac{2}{5}\right)_{2 m}\left(\frac{3}{5}\right)_{2 m}(1)_{2 m}}{(1)_{6 m}}$ & $q_{p}(5)-2 f_{p}$ \\
\hline 5 & $\frac{p-1}{5}$ & -1 & $-1,-1$ & $\frac{2^{8 m}\left(\frac{3}{2}\right)_{4 m}}{5^{5 m}\left(\frac{4}{5}\right)_{2 m}\left(\frac{6}{5}\right)_{2 m}}$ & $q_{p}(5)-3 f_{p}$ \\
\hline 5 & $\frac{3(p-1)}{10}$ & $-\frac{1}{16}$ & $\frac{3}{2}, \frac{3}{2}$ & $\frac{5^{5 m}\left(\frac{4}{5}\right)_{2 m}\left(\frac{6}{5}\right)_{2 m}(1)_{2 m}}{(2)_{6 m}}$ & $q_{p}(5)+2 f_{p}$ \\
\hline 5 & -1 & $-2,-2$ & $\frac{2^{8 m}\left(\frac{5}{2}\right)_{4 m}}{5^{5 m}\left(\frac{7}{5}\right)_{2 m}\left(\frac{8}{5}\right)_{2 m}}$ & $q_{p}(5)+3 f_{p}$ \\
\hline
\end{tabular}

Proof of the case $p \equiv 1(\bmod 5), m=\frac{p-1}{5}, x=-1, \alpha=\beta=-1$. By running twoFone we establish the identity

$$
{ }_{2} F_{1}\left[\begin{array}{ll}
-m, & 5 m+1 \\
4 m+\frac{3}{2}
\end{array} ;-\frac{1}{4}\right]=\frac{2^{8 m}\left(\frac{3}{2}\right)_{4 m}}{5^{5 m}\left(\frac{4}{5}\right)_{2 m}\left(\frac{6}{5}\right)_{2 m}} .
$$


From the basic properties of $\Gamma_{p}$, (8), and Lemma 1, we deduce

$$
\begin{aligned}
\frac{\left(\frac{3}{2}\right)_{4 m}}{\left(\frac{4}{5}\right)_{2 m}\left(\frac{6}{5}\right)_{2 m}} & =\frac{\left(\frac{1}{2}\right)_{\frac{5 m}{2}}\left(\frac{3}{2}+\frac{5 m}{2}\right)_{\frac{3 m}{2}}}{\left(\frac{4}{5}\right)_{2 m}\left(\frac{1}{5}\right)_{m}\left(\frac{6}{5}+m\right)_{m}} \\
& =\frac{\Gamma_{p}\left(\frac{1}{2}+\frac{5 m}{2}\right) \Gamma_{p}\left(\frac{3}{2}+4 m\right) \Gamma_{p}\left(\frac{4}{5}\right) \Gamma_{p}\left(\frac{1}{5}\right) \Gamma_{p}\left(\frac{6}{5}+m\right)}{\Gamma_{p}\left(\frac{1}{2}\right) \Gamma_{p}\left(\frac{3}{2}+\frac{5 m}{2}\right) \Gamma_{p}\left(\frac{4}{5}+2 m\right) \Gamma_{p}\left(\frac{1}{5}+m\right) \Gamma_{p}\left(\frac{6}{5}+2 m\right)} \\
& =\frac{\Gamma_{p}\left(\frac{7}{10}+\frac{4 p}{5}\right) \Gamma_{p}\left(\frac{4}{5}\right) \Gamma_{p}\left(\frac{1}{5}\right)}{\Gamma_{p}\left(\frac{1}{2}\right) \Gamma_{p}\left(\frac{2}{5}+\frac{2 p}{5}\right) \Gamma_{p}\left(\frac{4}{5}+\frac{2 p}{5}\right)} \\
& \equiv \frac{\Gamma_{p}\left(\frac{1}{5}\right) \Gamma_{p}\left(\frac{7}{10}\right)}{\Gamma_{p}\left(\frac{1}{2}\right) \Gamma_{p}\left(\frac{2}{5}\right)}\left(1+p\left(\frac{4}{5} H_{\frac{3(p-1)}{10}}-\frac{2}{5} H_{\frac{3(p-1)}{5}}-\frac{2}{5} H_{\frac{p-1}{5}}\right)\right) \\
& \equiv \frac{\Gamma_{p}\left(\frac{1}{5}\right) \Gamma_{p}\left(\frac{7}{10}\right)}{\Gamma_{p}\left(\frac{1}{2}\right) \Gamma_{p}\left(\frac{2}{5}\right)}\left(1+p\left(-\frac{8 q_{p}(2)}{5}+3 f_{p}\right)\right) .
\end{aligned}
$$

Since by the multiplication formula [11, p. 371],

$$
\Gamma_{p}\left(\frac{1}{5}\right) \Gamma_{p}\left(\frac{7}{10}\right)=\Gamma_{p}\left(\frac{2 / 5}{2}\right) \Gamma_{p}\left(\frac{2 / 5+1}{2}\right)=\frac{1}{2^{c}} \Gamma_{p}\left(\frac{1}{2}\right) \Gamma_{p}\left(\frac{2}{5}\right)
$$

it follows that

$$
\frac{\Gamma_{p}\left(\frac{1}{5}\right) \Gamma_{p}\left(\frac{7}{10}\right)}{\Gamma_{p}\left(\frac{1}{2}\right) \Gamma_{p}\left(\frac{2}{5}\right)} \equiv 4^{m}\left(1-\frac{2 p q_{p}(2)}{5}\right) \quad\left(\bmod p^{2}\right)
$$

Hence

$$
\begin{aligned}
{ }_{2} F_{1}\left[\begin{array}{rl}
-m, 5 m+1 & 5 m+\frac{1}{2} ;-\frac{1}{4}
\end{array}\right] & \equiv \frac{4^{p-1}}{5^{p-1}}\left(1-\frac{2 p q_{p}(2)}{5}\right)\left(1+p\left(-\frac{8 q_{p}(2)}{5}+3 f_{p}\right)\right) \\
& \equiv 1+p\left(-q_{p}(5)+3 f_{p}\right) \quad\left(\bmod p^{2}\right)
\end{aligned}
$$

and by recalling that $\beta=-1$ we are done.

Proof of the case $p \equiv 1(\bmod 5), m=\frac{3(p-1)}{10}, x=-\frac{1}{16}, \alpha=\beta=\frac{3}{2}$. Again by twoFone we have the identity

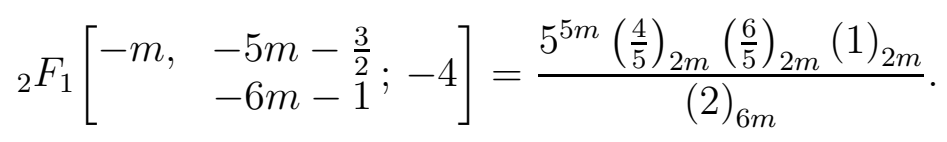


The basic properties of $\Gamma_{p}$, (88), and Lemma 1 imply

$$
\begin{aligned}
\frac{\left(\frac{4}{5}\right)_{2 m}\left(\frac{6}{5}\right)_{2 m}(1)_{2 m}}{(2)_{6 m}} & =\frac{\left(\frac{4}{5}\right)_{2 m}\left(\frac{1}{5}\right)_{\frac{2 m}{3}}\left(\frac{6}{5}+\frac{2 m}{3}\right)_{\frac{4 m}{3}}(1)_{2 m}}{(1)_{\frac{10 m}{3}}\left(2+\frac{10 m}{3}\right)_{\frac{8 m}{3}}} \\
& =\frac{\Gamma_{p}\left(\frac{4}{5}+2 m\right) \Gamma_{p}\left(\frac{1}{5}+\frac{2 m}{3}\right) \Gamma_{p}\left(\frac{6}{5}+2 m\right) \Gamma_{p}(1+2 m) \Gamma_{p}\left(2+\frac{10 m}{3}\right)}{\Gamma_{p}\left(\frac{4}{5}\right) \Gamma_{p}\left(\frac{1}{5}\right) \Gamma_{p}\left(\frac{6}{5}+\frac{2 m}{3}\right) \Gamma_{p}\left(1+\frac{10 m}{3}\right) \Gamma_{p}(2+6 m)} \\
& =\frac{\Gamma_{p}\left(\frac{1}{5}+\frac{3 p}{5}\right) \Gamma_{p}\left(\frac{3}{5}+\frac{3 p}{5}\right) \Gamma_{p}\left(\frac{2}{5}+\frac{3 p}{5}\right)}{\Gamma_{p}\left(\frac{4}{5}\right) \Gamma_{p}\left(\frac{1}{5}\right) \Gamma_{p}\left(\frac{1}{5}+\frac{9 p}{5}\right)} \\
& \equiv \frac{\Gamma_{p}\left(\frac{3}{5}\right) \Gamma_{p}\left(\frac{2}{5}\right)}{\Gamma_{p}\left(\frac{4}{5}\right) \Gamma_{p}\left(\frac{1}{5}\right)}\left(1+p\left(\frac{3}{5} H_{\frac{2(p-1)}{5}}+\frac{3}{5} H_{\frac{3(p-1)}{5}}-\frac{6}{5} H_{\frac{4(p-1)}{5}}\right)\right) \\
& \equiv \frac{\Gamma_{p}\left(\frac{3}{5}\right) \Gamma_{p}\left(\frac{2}{5}\right)}{\Gamma_{p}\left(\frac{4}{5}\right) \Gamma_{p}\left(\frac{1}{5}\right)}\left(1+3 p f_{p}\right) .
\end{aligned}
$$

By the reflection formula [11, (5) p. 369],

$$
\frac{\Gamma_{p}\left(\frac{3}{5}\right) \Gamma_{p}\left(\frac{2}{5}\right)}{\Gamma_{p}\left(\frac{4}{5}\right) \Gamma_{p}\left(\frac{1}{5}\right)}=1
$$

and therefore

$$
\begin{aligned}
{ }_{2} F_{1}\left[\begin{array}{rr}
-m, & -5 m-\frac{3}{2}
\end{array}-4\right] & \equiv 5^{\frac{3(p-1)}{2}}\left(1+3 p f_{p}\right) \equiv\left(1+p \frac{3 q_{p}(5)}{2}\right)\left(1+3 p f_{p}\right) \\
& \equiv\left(1+p\left(\frac{3 q_{p}(5)}{2}+3 f_{p}\right)\right) \quad\left(\bmod p^{2}\right) .
\end{aligned}
$$

The desired conclusion follows as soon as we note that $\beta=\frac{3}{2}$.

\section{Proof of the main theorem}

This opening lemma introduces two combinatorial identities.

Lemma 2. For any $1 \leq m \leq n$, and for any $z \in \mathbb{C}$,

$$
\left(1+\frac{1}{z}\right)^{n+1} \sum_{k=1}^{m} \frac{z^{k}}{k\left(\begin{array}{l}
n \\
k
\end{array}\right)}=H_{n}+H_{m}-H_{m^{\prime}}-\left(T_{n}(z)+T_{m}(1 / z)-T_{m^{\prime}}(z)\right)
$$

and

$$
\left(1+\frac{1}{z}\right)^{n+1} \sum_{k=m+1}^{n} \frac{z^{k}}{k\left(\begin{array}{l}
n \\
k
\end{array}\right)}=H_{n}+H_{m^{\prime}}-H_{m}-\left(T_{n}(1 / z)+T_{m^{\prime}}(z)-T_{m}(1 / z)\right)
$$

where $m^{\prime}=n-m$, and

$$
T_{r}(z)=\frac{1}{\left(\begin{array}{l}
n \\
r
\end{array}\right)} \sum_{k=0}^{r-1}\left(\begin{array}{l}
n \\
k
\end{array}\right) \frac{z^{k-r}}{k-r}
$$


Proof. After multiplying (11) by $z^{n}$, we find

$$
(z+1)^{n+1} \sum_{k=1}^{m} \frac{z^{k-1}}{k\left(\begin{array}{l}
n \\
k
\end{array}\right)}=\sum_{k=0}^{n-1}\left(\begin{array}{l}
n \\
k
\end{array}\right) \frac{z^{k}}{n-k}+\left(H_{n}+H_{m}-H_{n-m}\right) z^{n}-\frac{1}{\left(\begin{array}{c}
n \\
m
\end{array}\right)} \sum_{\substack{k=0 \\
k \neq m}}^{n}\left(\begin{array}{l}
n \\
k
\end{array}\right) \frac{z^{n+m-k}}{k-m} .
$$

Both sides of are polynomials of degree $n+m$, so it suffices to compare the coefficients of $z^{d}$ for each $d$ such that $0 \leq d \leq n+m$ :

$$
\sum_{k=1}^{m} \frac{\left(\begin{array}{c}
n+1 \\
d-k+1
\end{array}\right)}{k\left(\begin{array}{l}
n \\
k
\end{array}\right)}= \begin{cases}\frac{1}{n-d}\left(\left(\begin{array}{l}
n \\
d
\end{array}\right)-\frac{\left(\begin{array}{c}
n \\
n+m-d
\end{array}\right)}{\left(\begin{array}{l}
n \\
m
\end{array}\right)}\right) & \text { if } d \neq n \\
H_{n}+H_{m}-H_{n-m} & \text { if } d=n\end{cases}
$$

which can be easily verified by induction with respect to $m$. As regards (12), just subtract (11) from (11) where we set $m=n$.

Now we are able to show that the sum $S_{m}(x)$ is truly relevant to our needs.

Theorem 3. Let $p$ be an odd prime with $1 \leq m<\frac{p-1}{2}$. Let $x \in \mathbb{Z}_{p}$ be such that $x(1-4 x)$ is prime to $p$. Let

$$
Q_{p}(m, x)=H_{\frac{p-1}{2}}+H_{m}-H_{m^{\prime}}-\left(S_{\frac{p-1}{2}}(y)+S_{m}(x)-S_{m^{\prime}}(y)\right)
$$

where $m^{\prime}=\frac{p-1}{2}-m$ and $y=\frac{1}{16 x}$. Then

$$
\sum_{k=p-m}^{p-1}\left(\begin{array}{c}
2 k \\
k
\end{array}\right) x^{k} \equiv-\frac{2 p x Q_{p}(m, x)}{(1-4 x)^{\frac{p+1}{2}}} \quad\left(\bmod p^{2}\right)
$$

and

$$
\sum_{k=\frac{p+1}{2}}^{p-1-m^{\prime}}\left(\begin{array}{c}
2 k \\
k
\end{array}\right) y^{k} \equiv-\frac{2 p y Q_{p}(m, x)}{(1-4 y)^{\frac{p+1}{2}}} \quad\left(\bmod p^{2}\right) .
$$

Proof. We first note that for $1 \leq k \leq n=\frac{p-1}{2}$,

$$
\left(\begin{array}{l}
n \\
k
\end{array}\right) \equiv\left(\begin{array}{c}
2 k \\
k
\end{array}\right)(-4)^{-k}(\bmod p) \text {. }
$$

Hence for $z=\frac{1}{4 x}$, we find

$$
T_{r}(z) \equiv S_{r}\left(-\frac{1}{4 x}\right) \quad(\bmod p)
$$

and, by (11),

$$
(1-4 x)^{\frac{p+1}{2}} \sum_{k=1}^{m} \frac{(1 / x)^{k}}{k\left(\begin{array}{c}
2 k \\
k
\end{array}\right)} \equiv Q_{p}(x, m) \quad(\bmod p)
$$


Futhermore, for $1 \leq k \leq \frac{p-1}{2}$,

which imply

$$
k\left(\begin{array}{c}
2 k \\
k
\end{array}\right)\left(\begin{array}{c}
2(p-k) \\
p-k
\end{array}\right) \equiv-2 p \quad\left(\bmod p^{2}\right)
$$

$$
\sum_{k=p-m}^{p-1}\left(\begin{array}{c}
2 k \\
k
\end{array}\right) x^{k}=\sum_{k=1}^{m}\left(\begin{array}{c}
2(p-k) \\
p-k
\end{array}\right) x^{p-k} \equiv-2 p x^{p} \sum_{k=1}^{m} \frac{(1 / x)^{k}}{k\left(\begin{array}{c}
2 k \\
k
\end{array}\right)} \quad\left(\bmod p^{2}\right),
$$

and (14) follows immediately. In a similar way, by applying (12), we show (15).

Finally, all is set up for our final proof.

Proof of Theorem 1. If $p \equiv 1$ modulo 3, by (13), Lemma 1, (10) and Table 1,

$$
\begin{aligned}
Q_{p}\left(\frac{p-1}{3},-2\right) & =H_{\frac{p-1}{2}}+H_{\left\lfloor\frac{p}{3}\right\rfloor}-H_{\left\lfloor\frac{p}{6}\right\rfloor}-S_{\frac{p-1}{2}}\left(-\frac{1}{32}\right)-S_{\frac{p-1}{3}}(-2)+S_{\frac{p-1}{6}}\left(-\frac{1}{32}\right) \\
& \equiv-2 q_{p}(2)-\frac{3 q_{p}(3)}{2}+2 q_{p}(2)+\frac{3 q_{p}(3)}{2}-4 q_{p}(2)+q_{2}(p)-\frac{3 q_{p}(3)}{2}+\frac{3 q_{p}(3)}{2} \\
& \equiv-3 q_{p}(2) \quad(\bmod p) .
\end{aligned}
$$

Thus, by (14) and (15) together with (1) and (2), we obtain the first pair (15),

$$
\begin{aligned}
& \sum_{k=1}^{\left\lfloor\frac{2 p}{3}\right\rfloor}\left(\begin{array}{c}
2 k \\
k
\end{array}\right)(-2)^{k} \equiv \sum_{k=1}^{p-1}\left(\begin{array}{c}
2 k \\
k
\end{array}\right)(-2)^{k}-\frac{4 p Q_{p}\left(\frac{p-1}{3},-2\right)}{9^{\frac{p+1}{2}}} \equiv 0 \quad\left(\bmod p^{2}\right), \\
& \sum_{k=0}^{\left\lfloor\frac{5 p}{6}\right\rfloor} \frac{\left(\begin{array}{c}
2 k \\
k
\end{array}\right)}{(-32)^{k}} \equiv \sum_{k=0}^{\frac{p-1}{2}} \frac{\left(\begin{array}{c}
2 k \\
k
\end{array}\right)}{(-32)^{k}}+\frac{p Q_{p}\left(\frac{p-1}{3},-2\right)}{16(1+1 / 8)^{\frac{p+1}{2}}} \equiv\left(\frac{2}{p}\right) \quad\left(\bmod p^{2}\right) .
\end{aligned}
$$

Moreover, if $p \equiv 1$ modulo 5 then by (13), Lemma 1, (10) and Table 1,

$$
\begin{aligned}
Q_{p}\left(\frac{p-1}{5},-1\right)= & H_{\frac{p-1}{2}}+H_{\left\lfloor\frac{p}{5}\right\rfloor}-H_{\left\lfloor\frac{3 p}{10}\right\rfloor}-S_{\frac{p-1}{2}}\left(-\frac{1}{16}\right)-S_{\frac{p-1}{5}}(-1)+S_{\frac{3(p-1)}{10}}\left(-\frac{1}{16}\right) \\
\equiv & -2 q_{p}(2)-\frac{5 q_{p}(5)}{4}-\frac{5 f_{p}}{4}+2 q_{p}(2)+\frac{5 q_{p}(5)}{4}-\frac{15 f_{p}}{4} \\
& \quad-5 f_{p}-q_{p}(5)+3 f_{p}+q_{p}(5)+2 f_{p} \equiv-5 f_{p} \quad(\bmod p),
\end{aligned}
$$

and

$$
\begin{aligned}
Q_{p}\left(\frac{2(p-1)}{5},-1\right)= & H_{\frac{p-1}{2}}+H_{\left\lfloor\frac{2 p}{5}\right\rfloor}-H_{\left\lfloor\frac{p}{10}\right\rfloor}-S_{\frac{p-1}{2}}\left(-\frac{1}{16}\right)-S_{\frac{2(p-1)}{5}}(-1)+S_{\frac{p-1}{10}}\left(-\frac{1}{16}\right) \\
\equiv & -2 q_{p}(2)-\frac{5 q_{p}(5)}{4}+\frac{5 f_{p}}{4}+2 q_{p}(2)+\frac{5 q_{p}(5)}{4}+\frac{15 f_{p}}{4} \\
& -5 f_{p}-q_{p}(5)-3 f_{p}+q_{p}(5)-2 f_{p} \equiv-5 f_{p} \quad(\bmod p) .
\end{aligned}
$$

Then the second pair (66) and the third pair (17) come from (14), (15), (3) and (44).

Acknowledgments. The first author is funded by the Startup Foundation for Introducing Talent of Nanjing University of Information Science and Technology (2019r062). 


\section{REFERENCES}

[1] A. Adamchuk, Comments on OEIS A066796 in 2006, The On-Line Encyclopedia of Integer Sequences, http://oeis.org/A066796.

[2] S. B. Ekhad, Forty "strange" computer-discovered [and computer-proved(of course)] hypergeometric series evaluations, The Personal Journal of Ekhad and Zeilberger, http://www.math.rutgers.edu/ zeilberg/mamarim/mamarimhtml/strange.html, (2004).

[3] F. W. J. Olver, D. W. Lozier, R. F. Boisvert and C. W. Clark, NIST Handbook of Mathematical Functions, Cambridge University Press, 2010.

[4] E. Lehmer, On congruences involving Bernoulli numbers and the quotients of Fermat and Wilson, Ann. Math. 39 (1938), 350-360.

[5] M. Lerch, Zur Theorie des Fermatschen Quotienten $\left(a^{p-1}-1\right) / p=q(a)$, Math. Ann. 60 (1905), 471-490.

[6] G.-S. Mao, Proof of a conjecture of Adamchuk, preprint, arXiv:2003.09810v4.

[7] G.-S. Mao, On a supercongruence conjecture of Z.-W. Sun, preprint, arXiv:2003.14221v2.

[8] G.-S. Mao and H. Pan, p-adic analogues of hypergeometric identities, preprint, arXiv:1703.01215v4.

[9] S. Mattarei and R. Tauraso, Congruences for central binomial sums and finite polylogarithms, J. Number Theory 133 (2013), 131-157.

[10] H. Pan and Z.-W. Sun, Proof of three conjectures of congruences, Sci. China Math. 57 (2014), no. 10, 2091-2102.

[11] A. M. Robert, A course in p-adic analysis, Graduate Texts in Mathematics, 198. Springer-Verlag, New York, 2000.

[12] Z.-W. Sun, Fibonacci numbers modulo cubes of primes, Taiwanese J. Math. 17 (2013), no. 5, $1523-1543$.

[13] Z. W. Sun and R. Tauraso, New congruences for central binomial coefficients, Adv. in Appl. Math. 45 (2010) 125-148.

[14] H. S. Vandiver, Symmetric functions formed by systems of elements of a finite algebra and their connection with Fermat's quotient and Bernoulli's numbers, Ann. Math. 18 (1917) 105-114.

[15] H. C. Williams, A note on the Fibonacci quotient $F_{p-\varepsilon} / p$, Canad. Math. Bull. 25 (1982), 366-370.

[16] H. C. Williams, Some formulas concerning the fundamental unit of a real quadratic field, Discrete Math. 92 (1991), 431-440.

Department of Mathematics, Nanjing University of Information Science and TechNOlOGy, Nanjing 210044, People's Republic of China

E-mail address: maogsmath@163.com

Dipartimento di Matematica, Università di Roma "Tor Vergata", via della Ricerca Scientifica, 00133 Roma, Italy

E-mail address: tauraso@mat.uniroma2.it 